\title{
A PHARMACY CARER SUPPORT SERVICE: OBTAINING NEW INSIGHT INTO CARERS IN THE COMMUNITY
}

\begin{abstract}
\section{Objectives}

Unpaid carers have many and varied responsibilities in society, which can include medication management for the person they support. However, the potential for Australian community pharmacies to better assist carers is relatively unexplored. This mixed-methods study investigated the acceptability of a local carer support service by trained community pharmacy staff, including issues regarding the implementation and impact of this service.
\end{abstract}

\section{Methods}

Staff from 11 community pharmacies in South-East Queensland, Australia were trained to deliver a six-step carer support service between September 2016 and March 2017. Pharmacies were supported by a carer and pharmacist mentor pair and asked to recruit up to six carers each. Evaluations of staff training were descriptively analysed. Semi-structured interviews were undertaken with pharmacy staff and interview transcripts were analysed thematically.

\section{Key findings}

Staff training evaluations were positive; participants acquired new information about carers and rated the service highly in terms of its importance within the pharmacy setting. Feedback was obtained on how to improve the training, such as further opportunities for role-play. Seven 
staff members were interviewed and data analysis revealed two main themes: i) implementation of the carer support service, and ii) perceived impact on pharmacy staff.

Positive attitudes towards recognising and supporting carers, and training and mentoring, were identified with community pharmacies viewed as a suitable place for delivering this new service. New insights into the impact of caring were widely reported, which staff had not appreciated from previous carer interactions. Structural issues including space and time pressures, and a lack of awareness about the types of support currently available to carers were emphasised.

\section{Conclusion}

Pharmacy staff are well positioned to support carers. Engaging carers in conversation to better understand their needs is a small step with potential for big gains, including a more empathetic understanding of their individual circumstances and overall wellbeing. 


\section{INTRODUCTION}

Carers play an invaluable and essential role in society. When a person is cared for by an (unpaid) carer, such as a family member, this somewhat reduces the work of more formal healthcare services. While the most appropriate term for carers is contested, with some people not wanting to be defined as such, ${ }^{[1]}$ this term is used in this study to define a person who provides support, in whatever form, to another person with a chronic condition.

Carer burden, and self-neglect of a carer's own health needs, has been reported across a range of diverse contexts. ${ }^{[2-5]}$ Recent Australian studies demonstrated that many carers also experience chronic condition/s, including pain, arthritis and depression ${ }^{[6,7]}$ which may or may not be as a result of their caring role. There are 2.86 million self-reported carers in Australia. ${ }^{[8]}$ Twenty four per cent (115/481) of carers recently surveyed by Carers Queensland, a nongovernment support organisation, were dissatisfied with their quality of life, and expected it to deteriorate further over the next 12 months. ${ }^{[9]}$ Yet, many carers will continue caring because emotionally or logistically they feel they have little choice. Carer burden is multi-faceted, and both subjective, such as the perceived impact on mental health, and objective, including a worsening financial position and time expended in caring duties. ${ }^{[10]}$ Health professionals have an opportunity to work with carers to reduce this burden. Internationally, community pharmacy staff are well positioned in this regard as they have frequent contact with carers, e.g. when carers collect prescription medication.

Pharmacy staff may be well placed to support carers, particularly with respect to medication management. However, UK research has acknowledged that further effort is needed towards identifying and recording carer status and working with carers within the pharmacy setting. ${ }^{[11]}$ Clarification and understanding of legal responsibilities and professional obligations of 
community pharmacy staff regarding medication provision to carers, and the development of a carer card to streamline medication access on behalf of the care-receiver, were recommendations from Australian research. ${ }^{[6,12]}$ In one of the few community pharmacy studies to focus explicitly on carers, staff from 45 UK pharmacies were trained on the challenges that carers may face, the support they may require, and referral options, such as to the carer's General Practitioner (GP) or other support services. ${ }^{[13,14]}$ Discussions included how to identify and support carers in the pharmacy, e.g. offer a prescription medicine delivery service. ${ }^{[14]}$ Findings revealed the need to increase staff awareness of this population; training and professional support to encourage staff to engage with carers was recommended. ${ }^{[13]}$ The positive results from the UK study, ${ }^{[13]}$ and the need for community pharmacies to engage more directly with, and support, carers, led to the development of an extended support service within the Australian context. ${ }^{[15]}$

Given this was a new Australian service, the aim of this study was to evaluate the acceptability of the carer support service, from the perspective of trained pharmacy staff. The study objectives were to: i) adapt and extend the UK carer support service to fit the Australian community pharmacy model; ii) explore pharmacy staff perceptions of the training received for delivering this service; iii) assess the acceptability for delivering this service by community pharmacy staff. The authors expected the findings to inform them as to whether a larger trial of this new pharmacy service was warranted. This study provides new insight into the role that pharmacy staff can play towards better supporting carers, a relatively unrecognised and underserved population. Results pertaining to the carer participants, such as their opinions of the service and documented outcomes will be reported separately.

\section{METHOD}




\section{Study Design}

This mixed-methods study builds on and extends the UK work ${ }^{[13]}$ by incorporating a patientcentred support service adapted from an Australian pharmacy mental health study. ${ }^{[16]}$ The carer support service aimed for pharmacy staff (pharmacists or assistants trained in over-the-counter medicine use) to identify and build relationships with carers and explore and address their needs (as both a carer and consumer) over a three-month period or agreed time-frame. The UK carer training and promotional resources ${ }^{[14]}$ were adapted, with permission, to suit the Australian context. Materials showcased the lived experiences of Australian carers, e.g. videos of carer stories ${ }^{[17]}$ and links to relevant non-government organisations and support services, e.g. Carers Queensland, Lifeline Service Finder App; the research team focused on carer health and wellbeing. Half of the training was dedicated to informing the staff about what the pharmacy service involved, step-by-step, with reference to the carer documentation folder (Table 1). A representative from Carers Australia (Queensland branch), a young carer consultant with lived caring experience who was a previous young carer and carer ambassador for Carers Australia, and an advisor from the UK study provided feedback on material development. Minor changes were made, including removing the term 'carer burden' as carers may also experience positive benefits from their role.

Staff training was piloted with three pharmacy academics, one pharmacist practitioner and six pharmacy students. Feedback recommendations included providing further information on carer support services, increasing the number of participating pharmacies from six to ten and reducing the number of recruited carers per pharmacy from ten to six. Changes in sample sizes were suggested to enhance recruitment rates, noting the busyness of community pharmacies. Minimal changes were made to promotional materials and the carer documentation folder (see intervention details; Table 1), e.g. changing cultural background to ethnicity. Filmed interviews 
with two consultants with lived carer experiences were included as a training resource; answers to questions such as how can community pharmacy help a variety of carers and what services do Carers Queensland offer were provided.

\section{$\underline{\text { Intervention details }}$}

The carer intervention involved six key-steps which were designed to be completed in a sequential fashion (Table 1; Figure 1); it could only be delivered by a trained staff member. While the intervention was designed to be flexible, staff were advised that a similar service took an average of 110 minutes to deliver over a three-month period. ${ }^{[16]}$

After consenting to participate in the project (Step 1), information was obtained on the carer context, e.g. demographics, who the carer supported (the care-receiver), the associated caring roles and responsibilities, such as emotional and physical support, and who was involved in the carer and care-receiver's personal healthcare team, e.g. social worker, specialist physician. The carer's GP could, with the carer's consent, be contacted regarding their study participation (Step 2). Step 3 involved a carer health assessment, with measures including the EQ-5D-3L General Health Questionnaire, ${ }^{[18]}$ the Bakas Caregiving Outcomes Scale (approval for use obtained), ${ }^{[19]}$ a 3 -item health literacy screening tool ${ }^{[20]}$ and the quantity and frequency of medicines used by both the carer and care-receiver. In Step 4, the pharmacy staff member worked with the carer to: i) identify any concerns arising from the above assessments, which could be medication related, ii) explore carer expectations of the community pharmacy, and iii) set two goals and develop a support plan to meet these goals. A signed agreement to work together was also obtained. Step 5 involved individualised carer follow-ups as needed. Staff were asked to document what occurred at each follow-up (whether this was face-to-face, via email or phone) and any recommendations or suggestions made. The final step (6) involved an 
assessment of goal achievement, completion of the same Step 3 measures and a brief service evaluation, e.g. the service motivated me to take better care of my own health (Strongly AgreeStrongly Disagree).

\section{Procedure}

University Ethics approval was obtained (GU Ref No: 2016/014). The study was located in the South-East region of Queensland which extends from outer Brisbane to the Gold Coast; this was local to the research team and provided easy access for training and mentoring purposes (i.e. within a distance of 110 kilometres from the University). Community pharmacies were required to have a semi-private consultation area and provide a dose administration aid and/or prescription management service (i.e. prescription storage). Consent from the pharmacy owner/manager, and permission for two staff members to attend a training session, were also required for participation. The research team sought to include a range of different community pharmacies based on business model (e.g. banner groups, independent) and location/socioeconomic status. A variety of recruitment methods were used, including discussions with attendees at a national pharmacy conference, emailing study information to professional colleagues, and support from a professional pharmacy organisation.

Staff training occurred in September/October 2016 at each pharmacy $(n=11)$ or other mutually convenient location, e.g. a pharmacy head office or the researchers' University. An eleventh pharmacy was recruited for two reasons: i) a pharmacy was identified early in the study as experiencing recruitment difficulties, and ii) trained staff from one pharmacy also worked in another pharmacy that were interested in being involved; both of these pharmacies were located within the same shopping centre complex. Hence, we agreed to their participation. Additional training was provided upon request to three of the 11 pharmacies. All pharmacies were 
provided with promotional materials, e.g. postcards, badges and posters relating to National Carers Week, carer documentation folders, and Carers Queensland information (in English and/or other language). Advice was provided on how to recruit carer participants, e.g. start by approaching people known to be carers, utilise postcards as a conversation starter, and consider who may be carers in the pharmacy, e.g. someone picking up medication for someone else. A $\$ 50$ (AUD) gift voucher was provided to each of the two staff members per pharmacy that attended the training. For the pharmacies that had more than two staff members trained, the two $\$ 50$ vouchers were provided to the pharmacy owner or manager for their distribution.

Based on the success of the mental health study, ${ }^{[21]}$ a registered pharmacist with extensive professional experience, including pharmacy ownership, and a young carer consultant were recruited and trained as study mentors. The mentors worked in partnership and were tasked with visiting each pharmacy twice during the study period, making monthly follow-up phone calls and/or emails to discuss progress, answer queries and provide advice. Pharmacy owners or managers were remunerated $\$ 50$ (AUD) for each individually completed carer documentation folder.

\section{Data collection}

Participants were asked to provide training feedback in the form of a paper-based survey at training completion. Participants were asked to individually respond to 12 survey questions concerning the carer training using a five-point Likert scale which ranged from strongly agree to strongly disagree. Two additional questions were asked about participant confidence to speak to carers, and the importance of this service, within the pharmacy setting; a 0-10 scale was used $(0=$ not at all confident/important to $10=$ very confident/important $)$. All responses were collected at the end of each training session by the facilitator. 
Further in-depth information about the service was obtained via a short telephone or face-toface interview. All pharmacies were sent an email seeking interest from any trained pharmacy staff member to participate in an interview; follow-up phone calls were made to nonresponding pharmacies. Exploratory interview questions were developed with reference to the literature $^{[22-24]}$ and input from the research team and key stakeholders (Table 2). Interviews were undertaken by researchers not involved in training delivery. Consent was obtained for audio-recording and participants were provided with a \$20 (AUD) gift voucher.

\section{Data analysis}

Both quantitative and qualitative analysis was undertaken for this mixed-methods study.

\section{Quantitative Analysis}

Descriptive statistics were used to analyse feedback from training sessions; frequencies were calculated for each question using SPSS v22.

\section{Qualitative Analysis}

The first author (SM) transcribed all interviews to assist data familiarisation. Transcripts were quality checked by the interviewer (either MK or AS); minimal amendments were made. The first author then re-read all transcripts and coded the data with respect to the interview topics as outlined in the interview guide, i.e. thematic analysis. ${ }^{[25]}$ This involved the identification of similar codes across the entire data set, e.g. all information related to barriers to service was grouped together, same with service facilitators, etc. These codes were then further refined by identifying sub-themes within the initial codes, known as axial coding. ${ }^{[25]}$ Another author (HS) who was external to the interview process read and independently coded the transcripts to 
confirm the thematic analysis. The two researchers (SM and HS) met to discuss their respective analyses; no changes were required.

\section{RESULTS}

\section{Research Participants}

Thirty-nine staff from 11 pharmacies were trained; evaluations were obtained from 31 staff (79.5\%). Seven staff (five pharmacists, an intern and assistant) from five pharmacies were interviewed; five were female. The average interview duration was 32.39 minutes (range 20.2149.49); four interviews were conducted over the phone. Two participants represented pharmacies that were unable to recruit any carers. In total, 20 carers were recruited into the service by five of the 11 pharmacies.

\section{Training Feedback}

Evaluations of the training via the survey were overwhelmingly positive (Table 3). Participant confidence to deliver the carer support service was rated highly with a mean rating of 8.3 (range 7-10). The importance of providing the carer support service within the pharmacy setting was also emphasised (mean 9.5; range 6-10). Participants commonly described new knowledge acquired as greater awareness of carer diversity, identification of carers (and that some may choose not to disclose this status) and 'thinking about the carer as opposed to the cared (survey respondent \#21 - pharmacist). Participants suggested that training could be improved by providing examples of a greater variety of caring situations, further role-play opportunities, and information about the availability and accessibility of carer support such as Government funding.

\section{Interviews}


Data analysis revealed two main themes: i) implementation of the carer support service, and ii) perceived impact on pharmacy staff. Additional quotes to support the results are provided (Table 4); participant codes refer to the pharmacy and participant number, e.g. P1_01 refers to participant one from pharmacy one (the first pharmacy recruited).

\section{Implementing the support service}

\section{Approaching and recruiting carers}

The importance of establishing rapport and trust to approach, as well as engage, carers in conversation about their situation was emphasised, as was the altruistic nature of carers and their tendency to deflect any focus on them. It was important to demonstrate to a carer that being approached was not an assessment or judgement of their coping abilities, but more an acknowledgement of their important role/s and the burden of responsibility they assume:

I guess it's sort of getting into their heads that it's okay to have support and ask for help, whereas I think a lot of these people take... a substantial burden on board and don't actually ask for help unless you know it hits a critical sort of spot... (P1_01)

Individual personality traits influenced staff approaches; one participant volunteered that they struggled with social interactions and therefore found it more difficult to initiate a meaningful conversation with an unknown customer. Other participants experienced difficulty in steering conversations away from routine topics to introduce the carer support service to familiar customers. Regardless, an established and trusted relationship was viewed as instrumental in initiating conversations about the new service. Targeted recruitment was a common approach, with staff identifying known carers or consumers who outwardly signalled they might be in need of help: 
It's probably me picking people that I knew or like they exhibited, they were either in a, in a position where I felt like they look like they're at their wits end... (P5_01)

\section{Implementation barriers}

A general interest from carers about the support service was discussed, although this did not always translate into recruitment and active participation. Carer burden sometimes prevented participation, especially for carers 'tethered' to a care-receiver with $24 / 7$ needs (see Table 4, 'carer burden'). Differences in cultural norms worked against recruitment in some areas, for example, people of Asian ethnicity were expected to assume caring roles for elderly family members. Carers were generally viewed as stoic individuals:

'[They] either didn't feel like they should be asking for help or they felt like they didn't need

$$
\text { help' (P1_01). }
$$

Some carers were open to talking about their role, others less so:

...some people were really good about it, and you know, really open, and comfortable with the fact that that's what they were doing, but I don't think other people were really ready in their own head to recognise it... (P5_01)

Insufficient time to deliver the carer service as intended was a common complaint. Some participants struggled to recruit and/or maintain follow-ups during busy trading periods; others were preoccupied with store renovations and staff changes. One participant stated that carers 
often came into the pharmacy at peak times when there was limited capacity to approach them and explain the service. Difficulties were amplified when carers were visibly upset.

\section{Implementation facilitators}

Participants stressed the importance of providing a private space in the pharmacy, and sufficient time to explain and provide the carer support service. Allocating time and designating motivated staff to deliver the service minimised work-flow interruption:

I feel like the project is a one-on-one thing, so, you would only need to have enough staff to cover the store for one member to step out and I don't think that's very difficult to lose one staff member... (P6_02)

Flexibility in the workplace allowed follow-ups to be scheduled around the carer's timetable and availability. Involving all staff members in the study was an important principle. Such engagement was reported in one pharmacy where the study was discussed at a staff meeting to ensure everyone understood what was required of them, thereby reinforcing the manager's commitment. This pharmacy successfully recruited six carers, of which five completed the service. Participants generally agreed that everyone needed to be self-motivated and interested in delivering the carer service; pressuring a colleague was not viewed as appropriate. When asked about the type of pharmacy participants thought was best suited to provide the carer service, a quieter, forward-dispensing pharmacy or one with a consultation room for sensitive conversations, was emphasised. One participant recommended pharmacies in close proximity to hospitals for easy access by consumers and their carers following discharge. 
The carer documentation folders worked for some, but not all, participants. One participant provided in-depth feedback on the study tools (see Tables 1 and 4), reflecting on the usefulness of the folder to explore changes to a carer's life:

...that one [Bakas Caregiving Outcome Scale] really opened up the conversation. It was so difficult to go through though, because overwhelmingly...from the perspective of the carers I'd spoken to, it's been a negative experience in terms of how it [caring] has affected their lives... you know, they're stressed, they're run down, they have to take care of their mother all the time, their self-esteem's gone down... (P6_01)

This particular participant also sighed when talking about the time required for completing carer documentation and suggested this might have been easier in an online format. The potential for bias was raised when participants helped carers complete the service evaluation located in the carer folder, particularly with questions related to the performance of the pharmacy staff member. Another participant suggested that the goal setting section of the folder could be re-worded to clarify that goals were focused on the carers themselves, not the carereceiver. Goals, while not necessarily difficult to identify, were seen as challenging to achieve for carers, although supporting them in their attempts was perceived positively, such as providing accountability.

Carers Australia brochures were regarded as a particularly useful resource, even for carers not recruited into the study. The training, and the mentoring which continued for the entire study duration, was greatly appreciated and valued by participants: 
...honestly if the [mentors] weren't as helpful as they were, I don't think we would have cared as much as we do. Like the ability to actually have them on our side and have them as a resource... (P6_02)

Although follow-up phone calls by mentors were widely appreciated, they provided stress for staff who had not recruited many carers. One pharmacist confirmed that she had reassured another staff member that the study was as concerned with assessing study processes as it was with achieving recruitment targets.

\section{Impact of implementing the carer service on pharmacy staff}

Participants acknowledged that study involvement gave them a much better understanding of their customers who were also carers, and the supports available to carers, both within and outside the pharmacy setting:

... if anything I changed my mindset on, you know, the role of carers...I learnt a bit more about the role and also the support mechanisms that are available to carers in the community...I didn't know what Carers Australia actually did...(P1_01)

Focusing on carers was widely viewed as an area of pharmacy practice that had been overlooked, with attention traditionally placed on care-receivers. In the following quote a pharmacist explained how the study challenged her views about carer identity and communication style; she became more proactive in the way she addressed them:

...this young girl she's got really terminal cancer basically and her partner comes in and gets her medication all the time, and I thought well he's really a carer but you 
wouldn't really think of him like that, so, yeah I think it changed my, the way that I sort of spoke to them, like if she wasn't around you know I'd ask him how he was going with it all... (P5_01)

Establishing, and deepening, communication between pharmacy staff and carers was seen as a tangible outcome of the new service; one participant explained how a carer subsequently appeared more comfortable seeking help in their pharmacy. Participants agreed that the new service fitted well in the pharmacy setting; it was viewed as a beneficial health service with pharmacy staff seen as the most appropriate health professionals to deliver it.

When participants were asked how they thought pharmacies could help carers beyond medication supply, they mentioned professional services, such as dose administration aids and home deliveries, providing healthcare advice, and identifying whether further referral might be needed. Being a sounding board for carers and providing a listening ear was seen as integral to the role of pharmacy staff. One participant suggested that individual pharmacies could benefit from the appointment of a specific liaison person from Carers Australia to facilitate easier discussions about particular carers' needs. The same liaison person could then make follow-up contact and ensure the carer could access the resources they were entitled to, and needed.

\section{DISCUSSION}

This study explored the perceptions and experiences of community pharmacy staff involved in implementing a carer support service. Notably, staff obtained new knowledge and insights into the lives of many of their customers, even those with whom they had established relationships and were on first name terms. Participants also gained greater awareness of the impact of the carer role on personal wellbeing; this is particularly significant when pharmacy staff tended to 
target carers they knew in the pharmacy, raising the question that staff may not know their carer customers as well as they think they do. Pharmacy staff were understood to be ideally placed to acknowledge and listen to carers, thus providing opportunities for them to normalise and support the role of carers in communities. For example, the introduction of a carer service into the pharmacy promoted the important role that carers fulfil, as well as understanding that carers were valued persons in their own right, i.e. beyond their caring role.

This was a small study, with only a small proportion of the total staff trained subsequently interviewed. However, the interviews provided rich data on how pharmacists may provide support to this often hidden group of unpaid carers; there is the potential for pharmacists to identify caregivers who may not be known to GPs. While one area of Australia was targeted for pharmacy recruitment for mentor and training access, participants came from a range of pharmacies (e.g. independent or group) of varying sizes and locations. The timing of data collection may have influenced interview response rate and participant recall; interviews were not conducted immediately after service completion but delayed until all carer folder documentation had been completed. Staff perceptions of the service were limited to the carers that they interacted with; results may not be generalisable to all Australian carers. The risk of interviewer bias is possible as interviews were facilitated by members of the research team, one of whom has been a carer. However, interviews were conducted by experienced researchers who were not involved in the study training, and a standard interview guide was used. Furthermore, the potential for bias was reviewed during data analysis by an external researcher with qualitative research experience. As with any other form of qualitative research, there is a risk of self-reported bias; no formal observations of service delivery were undertaken. 
The benefits of providing a carer support service were articulated for pharmacy staff. Previous research suggests that continuing such a professional service may result in stronger pharmacistconsumer relationships. ${ }^{[26]}$ Staff were evidently surprised by the diverse range of carers and the negative impact of the caring role on people's lives and health. Survey tools were viewed as useful in helping to probe sensitive areas, and hence deepen relationships. Going forward, pharmacy staff can use these tools ${ }^{[18,19]}$ in practice to engage carers in conversations about their health, and to identify areas for further support. This is particularly important as the tools removed the need for questions such as 'how are you?' which usually result in socially desirable responses, e.g. 'I'm fine,' thus restricting conversation.

Carers frequently access pharmacies for medication-related tasks. Participants agreed that pharmacies were well placed to provide a targeted carer service, however, when asked what this could involve, responses focused on the delivery of services already available, such as dose administration aids. While increasing carer awareness of existing pharmacy services is a step in the right direction, the results from this study confirm that staff gained further information about other support services available to carers. Such knowledge would enable pharmacy staff to offer more extensive carer support, such as signposting carers to external organisations. Other research has demonstrated that carers may not know which services are available to them, ${ }^{[27-29]}$ and that pharmacists can fulfil an important role as information providers and/or care coordinators. ${ }^{[30]}$ Participants emphasised that information on key support organisations such as Carers Australia was under-utilised in practice, and that they only became aware of these agencies following training in this study. There is an opportunity for pharmacy staff to become more engaged with such organisations, for example, during National Carers Week. It would be useful to gauge awareness of such organisations amongst the wider population of Australian pharmacists to identify if this knowledge gap is a systemic issue. 
Some difficulties were experienced in terms of carer recruitment. Reasons included insufficient time, differences in cultural norms between Caucasian and Asian populations, and an assumption that support was unnecessary if carers had been in their roles for an extensive period of time, i.e. were seen as having confidence in their roles and responsibilities. ${ }^{[31]}$ Importantly, some people may not recognise themselves as a carer perhaps as a protective measure towards the care-receiver, e.g. to maintain a semblance of independence. ${ }^{[32]}$ The concern with using carer terminology in service promotion material was referred to by one participant (Table 4); promotional material avoiding such terms would be required to target the attention of hidden carers who do not identify as a carer. Furthermore, how pharmacy staff use this term in their interactions with pharmacy consumers' needs further consideration. Canadian researchers described a Caregiving Trajectory in which carers may only realise that they need help after an initial period of adjustment; ${ }^{[31]}$ finding out where a person is situated in their caring journey may help pharmacy staff offer appropriate and timely support. Overall, this research provided opportunities for pharmacy staff to confirm the importance of the role of carers and promote wider discussion in this area. In keeping with increased focus on other sensitive topics, such as mental health and drug addiction, comparable attention on the role of carers in the community is warranted.

As with the delivery of other professional pharmacy services, ${ }^{[22,33,34]}$ an optimal mix of space, time, staff engagement and resourcing is needed to deliver a new service. Future, or continued delivery of the carer support service would benefit from placing study tools and other study documentation on a digital platform to increase access and usability. In addition to face-to-face training which obtained a positive response from staff, the mentoring provided was generally successful in terms of maintaining staff engagement with the study. For example, some staff who were unsuccessful with carer recruitment, were nonetheless willing to discuss their 
individual experiences in an interview and remained positive about the project and with the support they received. This was not unexpected given success in this area in a previous study which informed the development of the carer service. ${ }^{[16]}$ As training alone is unlikely to translate into full-scale implementation, ${ }^{[33]}$ additional mentoring support should be considered in the short-term for pharmacy staff providing any new service. Further adjustments based on our findings are needed to optimise the current training and service prior to implementation in a pilot study which, if successful, will inform a clinical trial.

\section{CONCLUSION}

There is a unique opportunity for community pharmacy staff to better understand, and provide a targeted service to support, a core group of consumers: those who are in a caring role for others. Currently, there has been limited focus on carers within this primary care setting as attention is primarily placed on the care-receiver. This study provides important guidance for the future delivery of a professional pharmacy service that can help to normalise and support the carer role. Findings demonstrated that by building staff capacity via training and mentoring, greater understanding of a carer's needs and circumstances is possible, with increased awareness of their individual challenges and associated burdens. 


\section{REFERENCES}

1. Commonwealth of Australia. Inquiry into better support for carers: Parliament of Australia; 2009.

2. van Beusekom I, et al. Reported burden on informal caregivers of ICU survivors: a literature review. Crit Care 2016;20(16).

3. Cohen SA, et al. Psychosocial factors of caregiver burden in child caregivers: results from the new national study of caregiving. Health Qual Life Outcomes 2015;13(120).

4. May CR, et al. Experiences of long-term life-limiting conditions among patients and carers: what can we learn from a meta-review of systematic reviews of qualitative studies of chronic heart failure, chronic obstructive pulmonary disease and chronic kidney disease? BMJ Open 2016;6(10):e011694.

5. Figueiredo D, et al. Caring for relatives with chronic obstructive pulmonary disease: how does the disease severity impact on family carers? Aging Ment Health 2014;18(3):38593.

6. Wheeler AJ, et al. Exploring the health consumers' perspective on the burden of chronic illness and the role of community pharmacy in chronic illness management. Canberra: Pharmacy Guild of Australia; 2014.

7. Jowsey T, et al. Time to care? Health of informal older carers and time spent on health related activities: an Australian survey. BMC Public Health 2013;13(1):374.

8. Deloitte Access Economics, Carers Australia. The economic value of informal care in Australia in 2015; 2015.

9. Walbank S. Quality of Life Audit 2016 Report: Carers Queensland; 2016.

10. Flyckt L, et al. Determinants of subjective and objective burden of informal caregiving of patients with psychotic disorders. Int J Soc Psychiatry 2015;61(7):684-92. 
11. Smith F, et al. Carers and Pharmacists: Supporting family carers of older people in community pharmacy: a review of guidelines and qualitative study. Final report. Pharmacy Research UK, London, 2016.

12. McMillan SS. Improving care for people with chronic conditions and their carers: how can community pharmacy help? [Doctoral Thesis]: Griffith University; 2016.

13. Mooney G, Wigfield A. Carer-Friendly Pharmacy Pilot: Summary of the Evaluation Results. Carers Trust. Carers Trust, Circle, University of Leeds, 2015. https://professionals.carers.org/carer-friendly-pharmacy-pilot-evaluation (accessed 13 July 2017)

14. Carers Trust. Carer-friendly pharmacy resources. 2015.

https://professionals.carers.org/pharmacy-pilot-resources (accessed 5 May 2017)

15. McMillan SS, et al. Caring for carers in community pharmacy. Res Social Adm Pharm 2017;13(1):254-6.

16. McMillan SS, et al. The impact of a person-centred community pharmacy mental health medication support service on consumer outcomes. J Ment Health 2017:1-10.

17. SBS. Insight: Young Carers. Australia. 2013.

https://www.youtube.com/watch?v=lcgI7qyqHOQ (accessed 5 December 2017)

18. EUROQOL. The EQ-5D-5L and EQ-5D-3L. 2013. www.euroqol.org (accessed 13 July 2017)

19. Bakas T, et al. Psychometric testing of the revised 15-item Bakas Caregiving Outcomes Scale. Nurs Res 2006;55(5):346-55.

20. Chew LD, et al. Validation of screening questions for limited health literacy in a large VA outpatient population. J Gen Intern Med 2008;23(5):561-6. 
21. Wheeler A, et al. Exploring the role of community pharmacy in supporting mental health consumers and carers. Canberra: Australian Governement Department of Health and The Pharmacy Guild of Australia; 2015.

22. O'Reilly CL, et al. A feasibility study of community pharmacists performing depression screening services. Res Social Adm Pharm 2015;11(3):364-81.

23. Lowrie R, et al. Experiences of a community pharmacy service to support adherence and self-management in chronic heart failure. Int J Clin Pharm 2014;36(1):154-62.

24. Latif A, et al. Examination of England's New Medicine Service (NMS) of complex health care interventions in community pharmacy. Res Social Adm Pharm 2016;12(6):96689.

25. Braun V, Clarke V. Using thematic analysis in psychology. Qual Res Psychol 2006;3(2):77-101.

26. Lucas B, Blenkinsopp A. Community pharmacists' experience and perceptions of the New Medicines Service (NMS). Int J Pharm Pract 2015;23(6):399-406.

27. Wingham $\mathrm{J}$, et al. Needs of caregivers in heart failure management: A qualitative study. Chronic Illn 2015;11(4):304-19.

28. Essue BM, et al. Informal care and the self-management partnership: implications for Australian health policy and practice. Aust Health Rev 2010;34(4):414-22.

29. Ploeg J, et al. Managing multiple chronic conditions in the community: a Canadian qualitative study of the experiences of older adults, family caregivers and healthcare providers. BMC Geriatrics 2017;17(1):40.

30. Willemse E, et al. Do informal caregivers for elderly in the community use support measures? A qualitative study in five European countries. BMC Health Serv Res 2016;16(1):270. 
31. Williams A, et al. A Canadian qualitative study exploring the diversity of the experience of family caregivers of older adults with multiple chronic conditions using a social location perspective. Int J Equity Health 2016;15(1):40.

32. Carter SR, et al. The willingness of informal caregivers to assist their care-recipient to use Home Medicines Review. Health Expect 2013.

33. Marquis $\mathbf{J}$, et al. Exploring the implementation of a medication adherence programme by community pharmacists: a qualitative study. Int J Clin Pharm 2014;36(5):1014-22.

34. Hanes CA, et al. Clinical services for obstructive sleep apnea patients in pharmacies: the Australian experience. Int J Clin Pharm 2014;36(2):460-8. 
Table 1: Details of staff training and carer documentation folder

Learning outcomes from staff training

- Understand and discuss the various carer terms used

- Describe the challenges that carers face and the support they may require

- Identify carers and know the type of roles they undertake (e.g. medicines management for someone else)

- Describe how your pharmacy can support carers

- Approach and communicate with carers to encourage participation in the intervention

- Know where to refer carers to (with consent) locally for further support

- Understand how to deliver the support service in your pharmacy

Carer documentation folder (support service up to three months duration)

- Step 1: Consent process

- Step 2: Carer health status - information on the carer role/s, responsibilities, and support network.

- Step 3: Initial health review - this included a range of tools such as the EQ-5D General Health Questionnaire, ${ }^{[21]}$ the Bakas Caregiving Outcomes Scale, ${ }^{[15]}$ and a 3-item health literacy screening tool. ${ }^{[30]}$

- Step 4: Support plan - after reviewing responses to Steps 2 and 3, the pharmacy staff member (trained pharmacist or pharmacy assistant) worked with the carer to: i) identify any concerns, ii) explore carer expectations of the community pharmacy, and iii) set two goals (one specific to the person's support/caring role and one personal goal). A support plan was developed and signed agreement made for staff and carer to work together.

- Step 5: Ongoing support - follow-ups with the carer were individualised and occurred as needed either face-to-face or via phone/email. Pharmacy staff were required to document what occurred at each follow-up and any recommendations/suggestions made.

- Step 5: Final review - assessment of goal achievement, repeat of the measures in Step 3 and service evaluation using a 5-point Likert Scale. 


\section{Table 2: Interview Questions}

1. What changes or improvements do you feel the carers experienced from the service? What about the pharmacy?

2. Can you suggest aspects of the service that worked well?

3. Can you suggest aspects of the service that did not work so well? How can these aspects be improved?

4. How else can pharmacies help carers?

5. How did you find it approaching carers in the pharmacy about the service? Can you provide any insight/comments on what worked to obtain a positive response from carers when approached about the service?

6. How did carers generally respond to being approached? And to the service?

7. What are your thoughts on the documentation? Any suggested changes?

8. How did carers respond to the documentation?

9. What tools did you feel were most important or helpful for this service?

10. What barriers or challenges did you encounter while implementing the service? What were your experiences with implementing the service in the pharmacy?

11. How do you think these barriers could be overcome in the future?

12. What helped you to implement this service in the pharmacy? And why?

13. Do you have any specific comments about the mentoring you received (telephone or face-to-face)?

14. Now that you have delivered the service, what, if any changes, would you make to the training?

15. Is this a service you would want to continue in the pharmacy after the study? Why/why not? How well does this service fit in the pharmacy?

16. What specific type of pharmacy setting is most suited to offering this service? 


\begin{tabular}{|c|c|c|c|c|c|}
\hline & $\begin{array}{l}\text { SA } \\
(n)\end{array}$ & $\begin{array}{l}\mathrm{A} \\
(n)\end{array}$ & $\begin{array}{l}\mathrm{N} \\
(n)\end{array}$ & $\begin{array}{l}\mathrm{D} \\
(n)\end{array}$ & $\begin{array}{l}\mathrm{SD} \\
(n)\end{array}$ \\
\hline The training met my expectations & 22 & 7 & 1 & - & - \\
\hline $\begin{array}{l}\text { After this training I now feel I will be able to identify } \\
\text { possible carers }\end{array}$ & 19 & 12 & - & - & - \\
\hline $\begin{array}{l}\text { After this training I now feel I will be able to say the right } \\
\text { things to carers }\end{array}$ & 15 & 16 & - & - & - \\
\hline The content was organised and easy to follow & 24 & 7 & - & - & - \\
\hline The printed materials given to me were good and useful & 24 & 7 & - & - & - \\
\hline I learned everything the trainer said we would learn & 22 & 8 & 1 & - & - \\
\hline $\begin{array}{l}\text { There were plenty of opportunities for discussion with the } \\
\text { rest of the group }\end{array}$ & 26 & 5 & - & - & - \\
\hline $\begin{array}{l}\text { There was enough time to discuss the things we wanted to } \\
\text { talk about }\end{array}$ & 26 & 5 & - & - & - \\
\hline $\begin{array}{l}\text { I now better understand what services and support are } \\
\text { available to carers }\end{array}$ & 21 & 8 & 2 & - & - \\
\hline $\begin{array}{l}\text { I now better understand why carers could benefit from } \\
\text { being referred to their local carers' service }\end{array}$ & 22 & 7 & 2 & - & - \\
\hline $\begin{array}{l}\text { I better understand how a pharmacy carers service can } \\
\text { benefit carers }\end{array}$ & 23 & 7 & 1 & - & - \\
\hline $\begin{array}{l}\text { I understand how we will use the resources (posters, } \\
\text { leaflets and forms etc.) within our pharmacy }\end{array}$ & 23 & 7 & 1 & - & - \\
\hline
\end{tabular}


Service Implementation

Approach Well if you tell them if you're [carer] not healthy... if you're not in good health ... you won't be able to offer any help to anyone

Use tact else... they start to think, Oh okay...so there's no guilt involved (P5_02)

I think with anything you need to explain what the benefits are. So like if it's something that they [carer] see could be, have the potential to be valuable, then it's much easier to get a positive response for them, whereas, if you don't know what the benefits are or if you're not able to communicate the benefits then it's much more difficult to sign patients up. (P6_02)

Staff ...some people are significantly more charismatic than I am...I don't build rapport with people that quickly, I need a few personality different interactions and then slowly the relationship builds...I don't think I could have that conversation; at least not in any

traits depth you know...I feel like they could sit down and answer the questions but it would be a shallower sort of dialogue (P6_01)

Barriers to service

\section{Carer}

burden the phone because there's no one that can help me...(P5_01)

...he [carer] has the flip phone hanging on a neck chain and, I didn't realise that what sounded like a wind coming from his phone was actually his wife sleeping...so that if she woke up while he was out of the house...that she wouldn't have a panic

Culture ...it's the way it runs in the family, the young ones looks after the elderly, looks after their parents, they don't think it's like a burden or anything or that it's affecting them, that's what they should be doing (P4_01)

Facilitators a lot of people um, said ah that's such a good idea you know, and everyone knows someone, you know, they all say oh my cousin

Demand or my friend and, they look after who, and you know they could really benefit from it or, the idea of the program, even for people that it didn't really relate to, was really positive. (P5_01)

Motivated It's not like a job...It's input from your heart... it feels like counselling in a way because they're telling you things, you're trying staff to listen to them, and you're trying to make them feel positive and give them some energy to do something (P5_02)

Privacy ...I think the thing that what worked really well was, really important to sort of have like have a room where they're [carers] actually able to bear their soul (P6_02) 
Tools and support

Surveys

(1) me asking) how would you rate your health from 0-100, that can tell me a lot; especially cause 1 would see 40 and I'm like oh, okay, you know, what makes you say that? They'll open up about it. Whereas if I sat down and said how are you feeling today? You know, it's less quantifiable, like they don't feel forced, they do the generic social construct of, I feel fine but you force them to quantify it...(P6_01)

... alternatively if it was a computer I could have them fill that in, say at home... it would definitely take away a lot of the benefits, because going through it is when they'd open up and start telling me a lot more about their roles as a carer, so it was good, but it's also really time consuming, you know (P6_01)

Goal ...every single person I spoke to I'd have to admit they were stumped at that goals thing and it wasn't until I put suggestions setting forward did they, did they sort of understand what I was getting at... a lot of them would then say oh I would love her to be able to eat three meals a day, and I'm like no, no, no, no, we're talking about you, yeah so that was a challenge for them... (P2_01)

Mentors ...I felt I could pick up the phone and call them [mentors/research staff] any time, um I thought they were really good...they sent us resources...I definitely felt that we were supported... (P2_01)

Training ...if I hadn't of had that training I would have had no idea how to help them [carers]. (P5_01)

Promotional

material and

resources

...there was one lady she takes care of her husband who has a brain tumour. He's very forgetful, um, her exact words were "I'm not a carer; I just do what any good wife should do"... material specifically aimed at carers was never going to work for her... (P6_01)

\footnotetext{
Impact of service implementation

Greater ...it definitely sort of changes your opinion of what a carer actually is, doesn't necessarily mean your Blue Care [in-home, understanding aged-care provider] nurse that comes in with your 80 year old grandma kind of, kind of thing (P5_01)

and rapport

... what the carers project required us to do was to actually have a proper sit down with them at a level that we don't normally have time for...I have a much better understanding of the patients who participated... I now understand a lot more about what they do as a carer, and that was really helpful in terms of helping to build that extra level of rapport (P6_01)

...I was at the front counter and I said how are you going you know and we just had a chat, and then she said to me I can't believe I can just come in and talk to you (laughs)... and I went of course you can, she goes but I didn't get a script today, I didn't need a product...spending your time talking to me and asking me how I am and I said of course... we are your community pharmacy and she said thank you that means lot...(P2_01)
} 
Step 1 Identify, recruit and consent

Step 2 My Health Status: demographics, information about the healthcare team for carer and care-receiver

Step 3 Initial Health Review: information about the impact of caring, quality of life, medication management

Support Plan: identify two goals (as a consumer and

Step 4 carer) and strategies to meet these goals

Step 5 Ongoing support \& monitoring: follow-ups

Step 6

Final Health Review: goal assessment and service

Figure 1: Steps in the carer intervention 